\title{
Knockdown of FBP1 enhances radiosensitivity in prostate cancer cells by activating autophagy
}

\author{
X. R. LI' ${ }^{1}$, K. Q. ZHOU ${ }^{1}$, Z. YIN ${ }^{1}$, Y. L. GAO ${ }^{1}$, X. YANG ${ }^{2, *}$ \\ ${ }^{1}$ Department of Urology, The Second Xiangya Hospital, Central South University, Changsha 410011, China; ${ }^{2}$ Department of Oncology, The \\ Second Xiangya Hospital, Central South University, Changsha 410011, China
}

${ }^{*}$ Correspondence: yangxintiger@csu.edu.cn

Received August 7, 2019 / Accepted December 13, 2019

\begin{abstract}
For patients with clinically early-stage localized prostate cancer, radiotherapy is another treatment that can achieve radical treatment in addition to radical prostatectomy. Despite this, there are still a large number of patients with prostate cancer who have a biochemical recurrence after undergoing radiotherapy, or even clinical recurrence, leading to treatment failure. Although the expression of the fructose-1,6-bisphosphatase 1 (FBP1) gene has been found to be absent in various tumors and is associated with a poor prognosis in tumor patients. However, the expression and role of FBP1 in prostate cancer are not clear. The purpose of this study was to investigate the role and mechanism of FBP1 in the radiotherapy resistance of prostate cancer. By analyzing the microarray data of prostate cancer radiotherapy resistant cells and parental cells (GSE53902), we found that FBP1 expression in DU145 radiotherapy resistant cells was significantly higher than in the DU145 parental cells. In addition, we searched for the expression of FBP1 in 492 prostate cancer samples from TCGA and found that its expression in prostate cancer was significantly higher than that in normal tissues. Knockdown of FBP1 expression significantly inhibited the proliferation of prostate cancer cells, promoted DNA damage-mediated apoptosis, and enhanced the sensitivity of prostate cancer cells to radiotherapy. Further mechanism analysis revealed that FBP1 knockdown could activate autophagy mediated by the AMPK-mTOR signaling pathway, while inhibition of the AMPK-mTOR signaling pathway could reverse FBP1 knockdown-mediated autophagy and apoptosis, as well as radiosensitization. In conclusion, this study clarified that FBP1 is an oncogene in prostate cancer, and the main mechanism for knockdown of FBP1 to increase radiosensitivity is to enhance autophagy mediated by the AMPK-mTOR signaling pathway. Therefore, FBP1 may be a potential target for enhancing prostate cancer radiotherapy.
\end{abstract}

Key words: prostate cancer, radiotherapy resistance, fructose-1,6-bisphosphatase 1, autophagy, AMPK-mTOR signaling pathway

Prostate cancer (PCa) has the highest incidence among males in western developed countries and its mortality ranks second, behind only lung cancer, and is the second in cancer-related mortality [1]. With the development of the economy, changes in dietary structure, the increasing proportion of the elderly population, and the continuous improvement of medical standards, the incidence and detection rate of prostate cancer in China is also increasing [2]. From 2000 to 2011, both morbidity and mortality of prostate cancer are on the rise [3]. At present, the treatment of prostate cancer mainly includes surgical treatment (radical prostatectomy), radiation therapy, and endocrine therapy [4]. Radiation therapy is one of the most important treatments for clinically early-stage localized prostate cancer [5]. However, there are still many patients with prostate cancer who have a biochemical recurrence after undergoing radical radiotherapy, or even clinical recurrence, leading to treatment failure [6]. Therefore, how to improve the radiation sensitivity of prostate cancer cells, reduce the dose of radiation therapy, effectively kill tumor cells, and improve the efficacy of radiation therapy are still urgent problems for clinicians and researchers.

Fructose-1,6-bisphosphatase 1 (FBP1) is a key ratelimiting enzyme in the gluconeogenesis process. It has two subtypes, one and two, which are expressed in the liver and muscle. In the gluconeogenesis process, FBP1 can catalyze the conversion of fructose-1,6-diphosphate to fructose-6phosphate, which acts as a gluconeogenesis rate-limiting enzyme, inhibits FBP1 activity, and reduces endogenous glucose production [7]. The intracellular glucose metabolism state is closely related, and it cooperates with the rate-limiting enzyme 6-phosphate fructokinase (PFK) in glycolysis to 
determine the dynamic transformation between intracellular glucose, fructose and fructose 1,6-diphosphate [8]. Although the expression of the FBP1 gene has been found to be absent in various tumors and is associated with a poor prognosis in tumor patients [9]. However, the expression and role of FBP1 in prostate cancer is not clear.

By analyzing the chip data (GSE53902) in prostate cancer radiotherapy resistant cells and parental cells [8], we found that FBP1 expression was significantly higher in radiationresistant DU145 cells than that in the DU145 parental cell. In addition, we confirmed this observation for the expression of FBP1 in 492 PCa samples in TCGA and found that its expression in PCa was significantly higher than that in normal tissues. In combination with the above-mentioned chip data, FBP1 is not only highly expressed in prostate cancer, but also highly expressed in radiotherapy-resistant prostate cancer. Therefore, we suspect that FBP1 may be involved in the radiotherapy resistance of prostate cancer as a cancerpromoting gene. The purpose of this study was to investigate the role and mechanism of FBP1 in the radiotherapy resistance of prostate cancer.

\section{Materials and methods}

Human tissue. From September 2015 to September 2018, we collected tissue samples from patients undergoing radical prostatectomy combined with radiotherapy from the Department of Urology, the Second Xiangya Hospital. According to the patient's efficacy, the cases with complete or partial remission were classified as a radiotherapy-sensitive group, while the cases with disease stabilization and progression were classified as a radiotherapy-resistant group. A total of 3 patients with sensitive and 3 cases of radiotherapy-resistant prostate cancer were collected, and 3 cases of benign prostatic hyperplasia $(\mathrm{BPH})$ were collected as a control group. The study has been approved by the Ethics Committee of the Second Xiangya Hospital, and all the subjects in the study have signed informed consent.

Western blot. Tissue samples or treated cells were collected and $200 \mu \mathrm{l}$ of RIPA was added for 30 min incubation at room temperature. The supernatant was aspirated by centrifugation at $12,000 \times \mathrm{g}$ for $5 \mathrm{~min}$ at $4{ }^{\circ} \mathrm{C}$. The BCA kit was used to quantify the protein concentration. The final protein concentration of the sample was adjusted to $4 \mathrm{mg} /$ $\mathrm{ml}$ by RIPA after adding $5 \times$ reducing sample buffer. Protein samples were separated on 12\% SDS/PAGE and blotted on NC membranes. The membrane was completely immersed in 3\% BSA-TBST and shaken at room temperature for 30 min for blocking. The primary antibody was diluted with $3 \%$ BSA-TBST (FBP1, 1:500, cat no. 12842-1-AP, Proteintech, Wuhan, China; GAPDH, 1:2000, cat no. ab128915, Abcam, Shanghai, China; Phospho-AMPKa (Thr172), 1:1000, cat no. 2535, CST, Danvers, MA, USA; AMPKa, 1:1000, cat no. 2532, CST; Phospho-mTOR (Ser2448), 1:1000, cat no. 2971, CST; mTOR, 1:1000, cat no. 2972, CST; Beclin 1, 1:1000, cat no. ab210498, Abcam; LC3B, 1:1000, cat no. ab221794, Abcam; p62, 1:1000, cat no. ab155686, Abcam; $\gamma$-H2AX, $1: 1000$, cat no. ab81299, Abcam), for $10 \mathrm{~min}$ incubation at room temperature and at $4^{\circ} \mathrm{C}$ overnight. The membrane was washed with TBST 5 times, each time 3 minutes. The membrane was incubated with secondary antibody (goat anti-rabbit IgG $(\mathrm{H}+\mathrm{L}) \mathrm{HRP}, 1: 10000$, cat no. 7074S, CST) diluted with $5 \%$ skim milk-TBST, and gently shaken at room temperature for $40 \mathrm{~min}$. After washing with TBST 6 times, each time for 3 minutes, the membrane was incubated with the ECL for $3 \mathrm{~min}$, and the exposed film was scanned directly. The Total Lab Quant V11.5 (Newcastle upon Tyne, UK,) software was used to read the integrated optical density (IOD) value of the bands.

Cell culture and transfection. The prostate cancer cell lines PC-3 and DU145 were purchased from Icell Bioscience (Shanghai, China). The PC-3 cells were cultured in 1640 medium supplemented with $10 \%$ fetal bovine serum and cultured at $37^{\circ} \mathrm{C}$ under $5 \% \mathrm{CO}_{2}$. DU145 cells were selected from DMEM medium supplemented with $10 \%$ fetal bovine serum and cultured at $37^{\circ} \mathrm{C}$ under $5 \% \mathrm{CO}_{2}$.

For cell transfection, after overnight cell culture in a six-well plate, the cell confluence was approximately $60-70 \%$. The medium was removed and $2 \mathrm{ml}$ of serum-free fresh 1640 medium was added. $150 \mu \mathrm{l}$ of 1640 nutrient diluted $10 \mu \mathrm{l}$ Lipofectamine 2000 was added to each well and incubated for $5 \mathrm{~min}$ at room temperature. $5 \mu \mathrm{l}$ of siRNA-1, siRNA-2, siRNA-scramble, and $150 \mu \mathrm{l}$ of 1640 medium were added to each well and mix gently. The diluted Lipofectamine 2000 was gently mixed with the diluted siRNA and allowed to stand at room temperature for $20 \mathrm{~min}$. The siRNA-transfection reagent mixture was added to the wells containing the cells and the culture solution, and the well plates were gently shaken to mix. After $6 \mathrm{~h}$, the medium containing the transfection reagent was discarded and replaced with a serum-containing medium. The cells were cultured in a $\mathrm{CO}_{2}$ incubator at $37^{\circ} \mathrm{C}$, and the transfection efficiency was measured by fluorescence microscopy $48 \mathrm{~h}$ later.

MTT. Each group of prostate cancer cells $\left(5 \times 10^{3}\right.$ in each well) was seeded into a 96-well plate, and $200 \mu$ l of the medium was added to each well. After the cells were attached to the wall, the True Beam electron linac X-ray was used for radiation treatment with different doses (6 MV X-rays at a dose gradient of $2 \mathrm{~Gy}, 4 \mathrm{~Gy}$, and $6 \mathrm{~Gy}$ ). One plate was not irradiated and served as a control group. After the radiation, cells were further cultured at $37^{\circ} \mathrm{C}$ and $5 \% \mathrm{CO}_{2}$. After $48 \mathrm{~h}$ culture, $20 \mu \mathrm{l}$ of MTT solution $(5 \mathrm{mg} / \mathrm{ml})$ was added into each well and incubated for 4 hours. The culture medium was carefully aspirated from each well, and $150 \mu \mathrm{l}$ of DMSO was added to dissolve the crystals completely. The absorbance at $570 \mathrm{~nm}$ of each well was measured by a microplate reader (MK3, Thermo).

Clonal formation. After cell transfection and irradiation, the PC-3 and DU145 cells were trypsinized and the individual cell suspension was prepared for seeding into a 
$60 \mathrm{~mm}$ culture dish at the density of 1000 cells/dish. The cells were cultured for $1-2$ weeks at $37^{\circ} \mathrm{C} 5 \% \mathrm{CO}_{2}$ in an incubator. When macroscopic clones appeared in the culture dish, the culture was terminated. The supernatant was discarded and washed 3 times with PBS. The cells were fixed with $1 \mathrm{ml}$ of $4 \%$ paraformaldehyde for 15 minutes and then incubated with crystal violet for $20 \mathrm{~min}$. After wash with PBS 3 times, the clones were counted directly in a microscope (low magnification). Finally, the clone formation rate was calculated as: (number of clones / number of cells inoculated) $\times 100 \%$.

Apoptosis assay. Apoptosis was measured by Annexin V-FITC/PI double staining apoptosis assay kit (cat no. 640914, Biolegend). After transfection with FBP1 siRNA and 2 Gy for 48 hours, the cells were digested with trypsin without EDTA and centrifuged at $1,500 \times \mathrm{g}$ for $5 \mathrm{~min} .500 \mu \mathrm{l}$ of Binding Buffer was added to resuspend the cells and Annexin V-FITC $5 \mu \mathrm{l}$ was added. After mixing, $5 \mu \mathrm{l}$ of Propidium Iodide was added and incubated at room temperature in the dark for $15 \mathrm{~min}$. Apoptotic cells were detected by flow cytometry (CytoFlex, Beckman).

Immunofluorescence staining. After cell transfection, the cells were digested, counted, and cultured overnight in a 12 -well plate containing cell slides. The medium of each well was aspirated, and the cells were washed 3 times with PBS for $5 \mathrm{~min}$ each time. The cells were fixed with $4 \%$ paraformaldehyde at room temperature for 20 minutes. $200 \mu \mathrm{l}$ of $0.5 \%$ Triton X-100 was added to the wells for $20 \mathrm{~min}$ at room temperature. The cells were blocked with goat serum for $60 \mathrm{~min}$. Primary antibody $(\gamma-\mathrm{H} 2 \mathrm{AX}$, diluted 1:250, cat no. ab81299, Abcam) was added to each well and incubated overnight in a wet box at $4{ }^{\circ} \mathrm{C}$. Secondary antibody (goat anti-rabbit IgG H\&L (FITC), diluted 1:1000, cat no. ab6717, Abcam) was added to the well and incubated for 1 hour at room temperature in the dark. DAPI was added dropwise to the well and incubated for 4 min in the dark. The fluorescence staining was observed and photographed under the fluorescence microscope.

Autophagosome observation. After transfection, the cells were seeded into 24 -well plates and cultured until the cell fusion degree was $50-70 \%$. The cells were infected with GFP-LC3 Adenovirus (MOI = 40, Genomeditech, Shanghai, China) and the virus infection efficiency was observed under the microscope after $48 \mathrm{~h}$. Then, the cells were subjected to $\mathrm{X}$-ray irradiation treatment at an irradiation dose of $2 \mathrm{~Gy}$, and the intracellular autophagosome was observed under a fluorescence microscope at 48 hours.

Statistical analysis. All statistical analyses were performed using GraphPad Prism (version 7.04). Continuity variables are expressed as mean \pm standard deviation. Student's $t$ test was used for comparison between the two groups; one-way ANOVA was used for three or more comparisons, and if there were differences between groups, the Tukey method was used for post hoc comparison. All statistical analyses were performed using a two-sided test, $\mathrm{p}<0.05$ indicating that the difference was statistically significant.

\section{Results}

The expression of FBP1 in prostate cancer. By searching NCBI GEO database, we obtained GSE53902 expression profile, including three parental DU145 cells (GSM1303309, GSM1303310, GSM1303311) and radiotherapy-resistant DU145 cells (GSM1303318, GSM1303319, GSM1303320). Using the GEO2R analysis in the GEO database, we found that FBP1 expression levels were significantly higher in the radiation-resistant cells than in the parental cells, approximately 5.48 times that of the parent (Figure 1A). In addition, we further verified the expression of FBP1 in prostate cancer in the GEPIA (Gene Expression Profiling Interactive Analysis) database and found that the expression level of FBP1 in prostate cancer was significantly higher than in normal tissues (Figure 1B). We collected tissue samples from patients undergoing radical prostatectomy combined with radiotherapy. We collected 3 cases of sensitive, 3 cases of radiotherapy-resistant prostate cancer, and 3 cases of benign prostatic hyperplasia (BPH) as a control group. The expression of FBP1 in prostate cancer tissues was detected by western blot. In prostate cancer tissues, the expression level of FBP1 was significantly higher than that of prostate proliferative tissue. At the same time, the expression level of FBP1 in the radiotherapy-resistant prostate cancer tissues was significantly higher than that in the radiotherapy-sensitive patients (Figure 1C). These results suggest that FBP1 may play an oncogenic role in prostate cancer.

Effect of FBP1 knockdown on radiosensitivity of prostate cancer. We analyzed the expression of FBP1 in three prostate cancer cell lines LNCaP, PC-3, and DU145 by western blot. FBP1 was expressed in all three cell lines, and the expression level of FBP1 was significantly higher in PC-3 and DU145 cells than in LNCaP (Figure 1D). We selected PC-3 and DU145 cells to study the function of FBP1. To investigate the effect of FBP1 on the radiosensitivity of prostate cancer, we interfered with the expression of FBP1 in PC-3 and DU145 cells by transfecting siRNA. We designed two pairs of specific siRNAs, named siRNA-1 and siRNA-2, and transfected them into PC-3 and DU145 cells, respectively. Finally, the expression level of FBP1 was detected by western blot. As shown in Figure 2A, after transfecting FBP1 siRNAs into two cell lines, the expression level of FBP1 was significantly lower than that of the scramble control group, and the siRNA-1 interference efficiency was better than that of siRNA-2. We used siRNA-1 for subsequent transfection experiments.

The prostate cancer cells transfected with FBP1 siRNA were treated with different doses of radiation for $48 \mathrm{~h}$, and the survival of prostate cancer cells was detected by MTT assay. The cell survival rate of PC-3 and DU145 decreased with the increase of radiation dose. Under the same radiation dose, the cell survival rate of the FBP1 interference group was significantly lower than that of the control group (Figure 2B). This result suggests that inhibition of the FBP1 


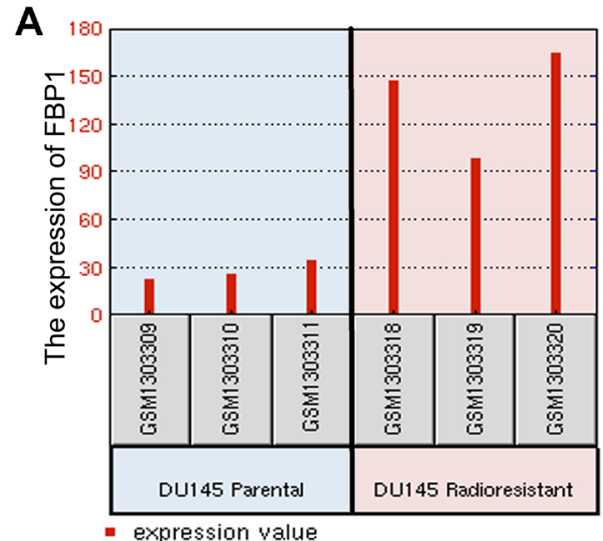

\section{C}
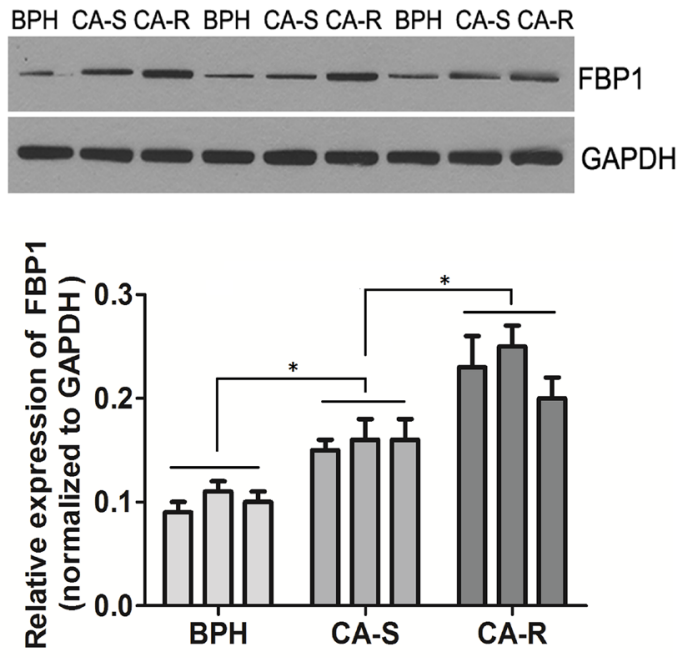

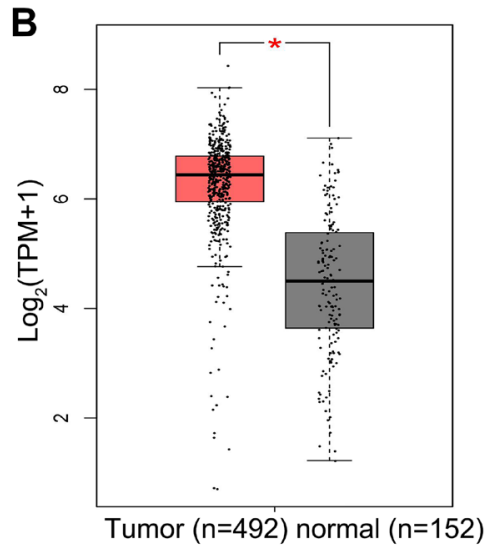

D
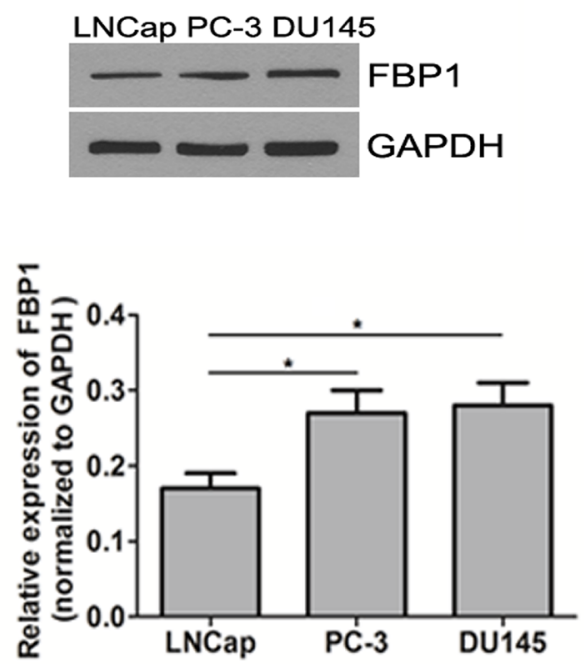

Figure 1. The expression of FBP1 in prostate cancer. A) The expression of FBP1 in GSE53902 expression profile, including three parental DU145 cells (GSM1303309, GSM1303310, GSM1303311) and radiation-resistant DU145 cells (GSM1303318, GSM1303319, GSM1303320). B) The expression of FBP1 in prostate cancer in the GEPIA (Gene Expression Profiling Interactive Analysis) database. Red color stands for the tumor group, grey stands for the normal group. TPM, Transcripts Per Million. C) The expression of FBP1 in prostate cancer tissue, including 3 cases of sensitive, 3 cases of radiotherapy-resistant prostate cancer, and 3 cases of benign prostatic hyperplasia (BPH) as a control group. D) The expression of FBP1 in prostate cancer cells. ${ }^{*} \mathrm{p}<0.05$. All experiments were repeated at least three times.

expression enhances the sensitivity of prostate cancer cells to radiation treatment.

We used the plate cloning assay to assess the effect of FBP1 on radiation-induced cell death. The number of cell clones decreased gradually with the increase of the dose of the reflection line in PC-3 and DU145 cells. Under the same radiation dose, the number of colonies in the FBP1 interference group was significantly lower than that in the control group (Figure 2C). This result suggests that inhibition of FBP1 expression can inhibit the ability of cell clone formation, thereby enhancing the sensitivity of prostate cancer cells to radiation treatment.
Radiation generates DNA-damaging oxygen free radicals by ionization, causing DNA damage, thereby killing tumor cells. Histone $\mathrm{H} 2 \mathrm{AX}$ has the function of responding to DNA molecular damage. When the body is exposed to radiation, DSBs are produced. H2AX 139 serine residue can be rapidly phosphorylated to form $\gamma$-H2AX. The amount of foci formed by $\gamma-\mathrm{H} 2 \mathrm{AX}$ in the nucleus is proportional to the number of DSBs in the nucleus and can be used to indicate DNA damage. We transfected prostate cells with FBP1 siRNA and irradiated with $2 \mathrm{~Gy}$ for $48 \mathrm{~h}$. Then, IF and western blot were performed to detect $\gamma-\mathrm{H} 2 \mathrm{AX}$ expression. In the FBP1 siRNA group, the number of $\gamma$-H2AX foci and the expression of $\gamma$-H2AX were 
A

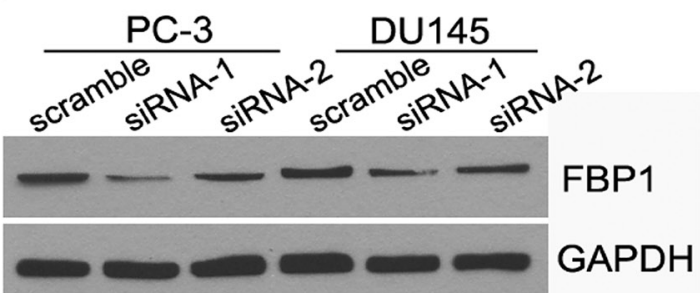

B

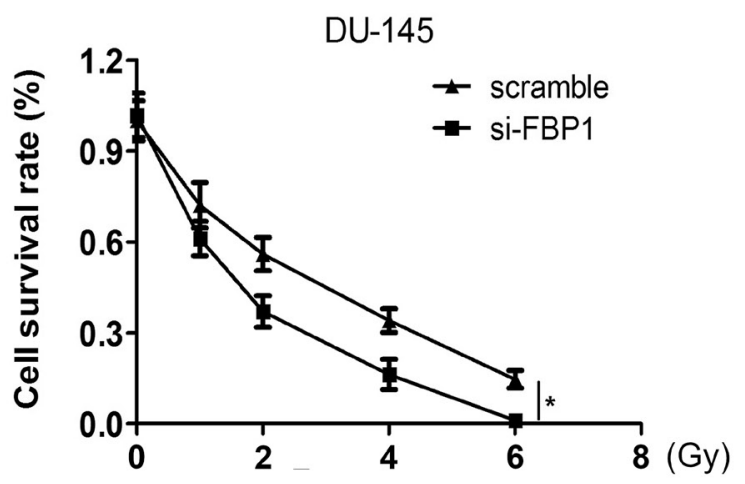

C
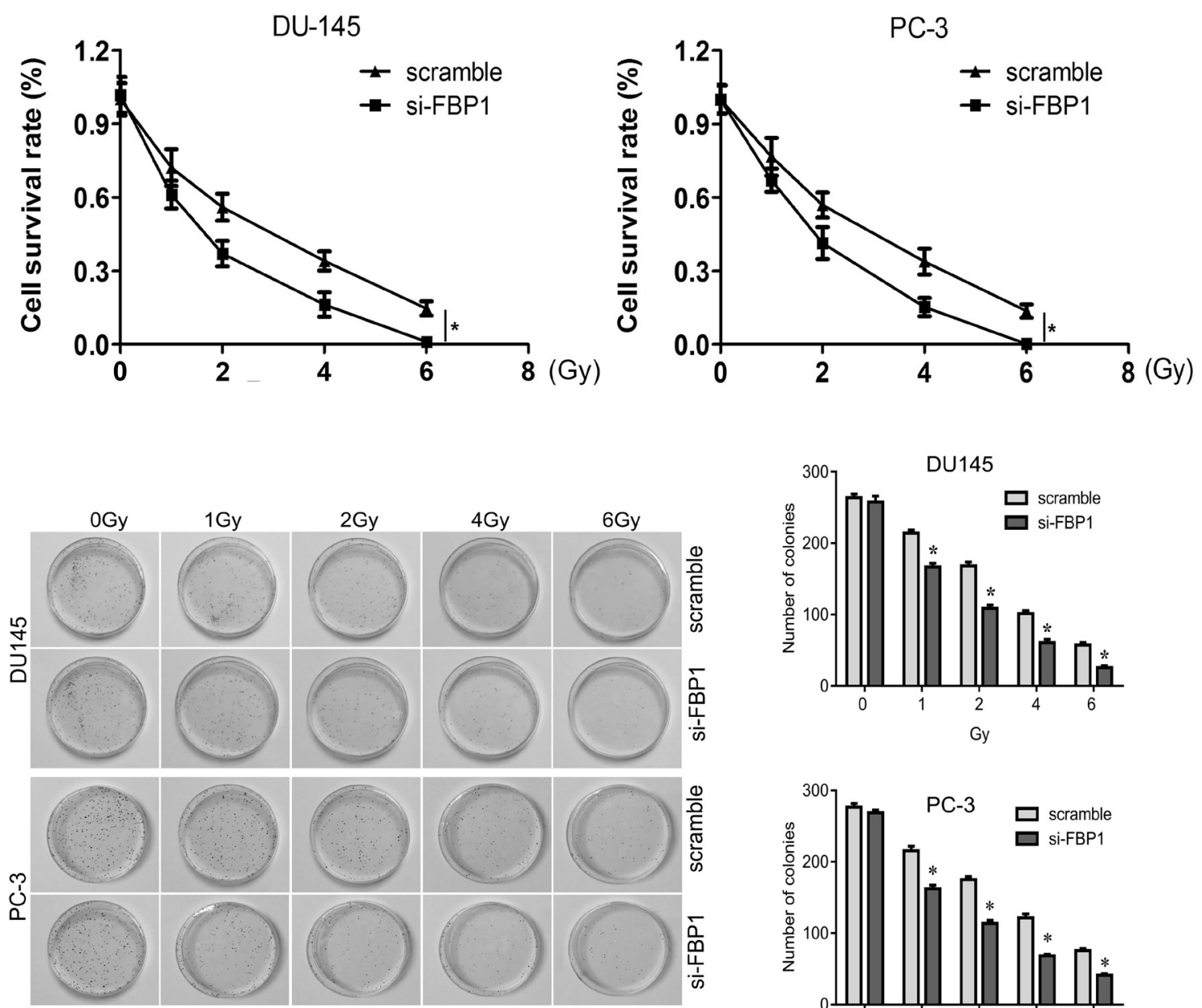
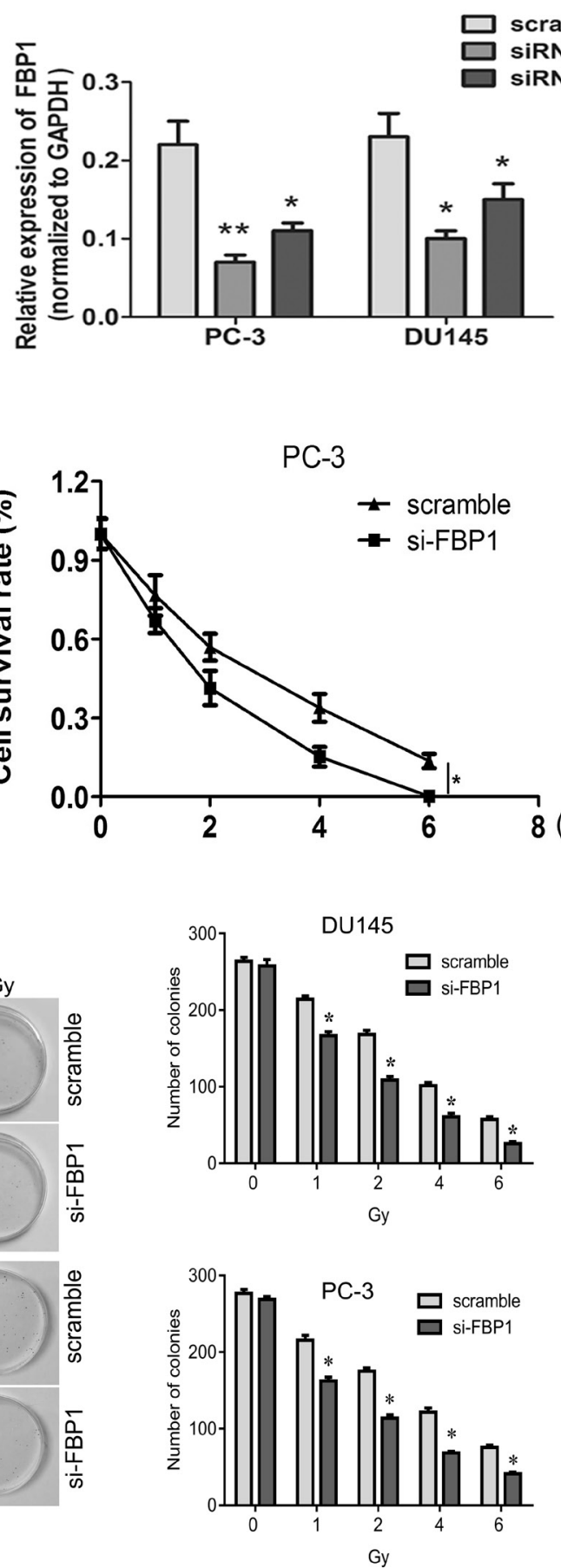

Figure 2. Effect of FBP1 knockdown on radiosensitivity of prostate cancer. A) The expression of FBP1 in prostate cancer cells after siRNA transfection. B) MTT was performed to measure cell viability after siRNA transfection and radiation. C) The clonal formation was performed to measure cell proliferation after siRNA transfection and radiation. ${ }^{*} \mathrm{p}<0.05$. All experiments were repeated at least three times.

significantly increased compared with the scramble group (Figures 3A, 3B). The results indicate that inhibition of FBP1 expression can aggravate the degree of DNA damage in prostate cancer cells after radiation exposure, and thus enhance the sensitivity of cells to radiation.
In addition, we transfected prostate cells with FBP1 siRNA, irradiated with $2 \mathrm{~Gy}$ doses of radiation, and $48 \mathrm{~h}$ later, the apoptosis of prostate cancer cells was detected by flow cytometry. After radiation exposure, PC-3 and DU145 cells showed significant apoptosis, while the apoptosis rate of the 
FBP1 interference group was significantly higher than that of the scramble group (Figure 3C). The results indicate that the expression of FBP1 can promote the apoptosis of prostate cancer cells after radiation exposure and enhance the sensitivity of cells to radiation.
Effect of FBP1 on autophagy of prostate cancer cells. In prostate cancer, it has been reported that activation of the autophagy pathway can increase the radiosensitivity of cells to radiation [10]. Therefore, in this study, we performed a corresponding test for autophagy in prostate cancer after

Figure 3. Effect of FBP1 knockdown on prostate cancer cell apoptosis. A) Immunofluorescence staining was performed to determine the DNA damage $(\gamma-\mathrm{H} 2 \mathrm{AX})$ after siRNA transfection and radiation. Magnification $400 \times$; bar $100 \mu \mathrm{m}$. B) The expression of $\gamma$ - $\mathrm{H} 2 \mathrm{AX}$ in prostate cancer cells after siRNA transfection and radiation. C) The flow cytometry was performed to measure the cell apoptosis after siRNA transfection and radiation. ${ }^{\star} \mathrm{p}<0.05$; ${ }^{* *} \mathrm{p}<0.01$. All experiments were repeated at least three times. 
interference with FBP1. When GFP-LC3B adenovirus infects cells, under normal conditions, GFP-LC3B can be observed to diffuse more uniformly in the cytoplasm under fluorescence microscope; when autophagy occurs, GFP-LC3B accumulates on the autophagosomal membrane. Fluorescent spots (punctate) were observed under a fluorescence microscope. In this study, after 2 Gy treatment of PC-3 and DU145 cells, obvious fluorescent spots were observed in the cells. At the same time, in the FBP1 interference group, the fluorescent spots in the cells were significantly higher than those in the control group (Figure 4A). In addition, FBP1 knockdown increased the expression level of autophagy substrate Beclin1, promoted the conversion of LC3B from type I to type II, and inhibited the expression of p62 (Figure 4B). This result indicates that autophagy occurs in cells after radiation treatment and can be enhanced by the inhibition of FBP1.

In order to explore the potential molecular mechanism of FBP1-mediated radioresistance of prostate cancer, we based on GSE53902 microarray expression data, significant differential gene expression between DU145 parental cells and radiation-resistant cells (Fold Change $>2$ and $\mathrm{p}<0.05$ ). By searching the KEGG database, Fisher's exact test and chi-square test were used to analyze the pathway of differ- 
ential gene participation, and $\mathrm{p}<0.05$ was set to obtain a significant difference. We found that FBP1 is involved in the AMPK (Adenosine 5'-monophosphate (AMP)-activated protein kinase) signaling pathway (Figure 4C). Many studies have reported that the AMPK signaling pathway is involved in the regulation of autophagy. Western blot analysis showed that knocking down FBP1 did not change the total protein of AMPK and mTOR but increased the expression of phosphorylated AMPK and decreased the expression of phosphorylated mTOR (Figure 4D). This result indicates that the AMPK-mTOR signaling pathway can be activated by inhibition of FBP1.

To further demonstrate whether FBP1 acts through the AMPK-mTOR signaling pathway, we interfered with the expression of FBP1 and co-treated prostate cancer cells with the AMPK-mTOR signaling pathway inhibitor compound C. The results indicate that compound $\mathrm{C}$ treatment significantly reversed FBP1 siRNA-mediated proliferation inhibi-
A

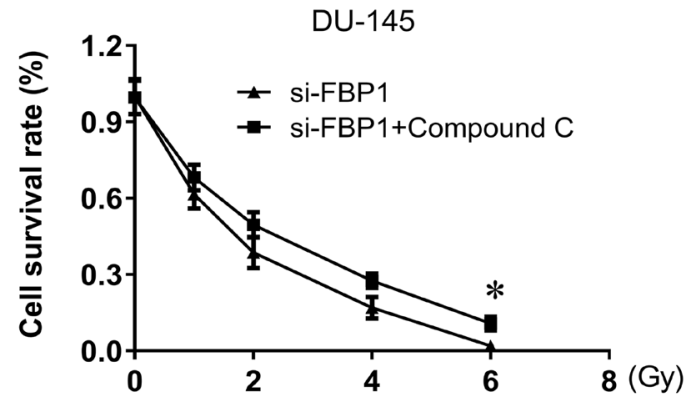

B

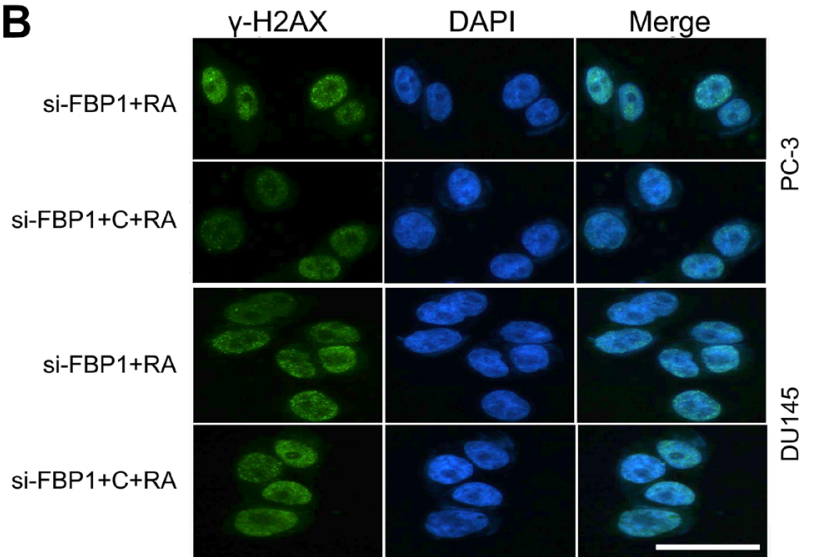

C

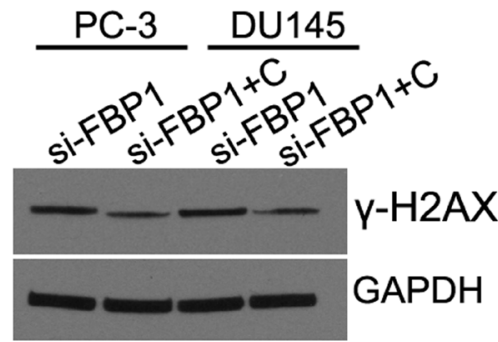

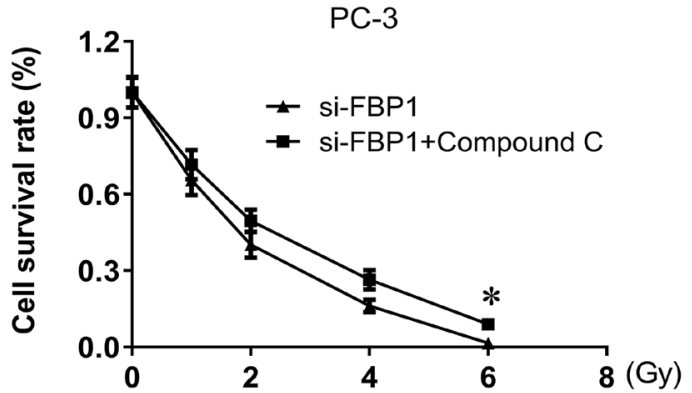

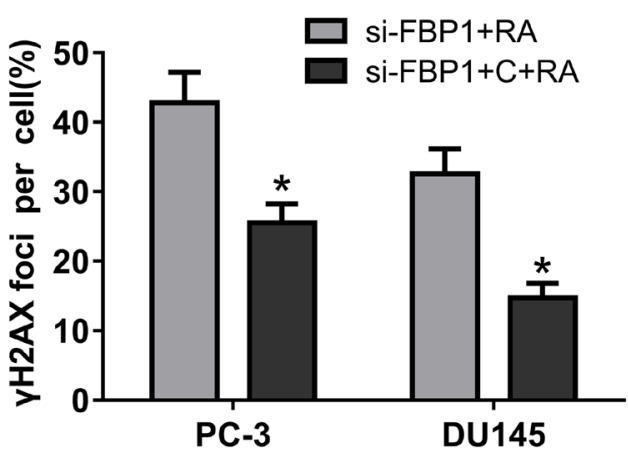

D

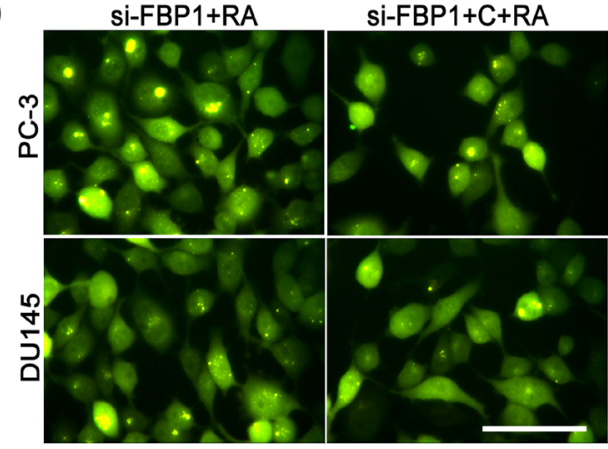


tion (Figure 5A), reduced FBP1 siRNA-mediated increase in $\gamma-\mathrm{H} 2 \mathrm{AX}$ foci and $\gamma-\mathrm{H} 2 \mathrm{AX}$ protein expression (Figures $5 \mathrm{~B}$, $5 \mathrm{C}$ ), and fluorescent spots (punctate) that inhibit GFP-LC3B formation (Figure 5D). These results suggest that FBP1 exerts an oncogenic effect through the AMPK-mTOR signaling pathway.

\section{Discussion}

In this study, we analyzed the NCBI GEO database to find a significant increase in FBP1 in prostate cancer cells resistant to radiation therapy and validated this result in prostate cancer tissues and cells. Further functional experiments demonstrated that the knockdown of FBP1 expression significantly inhibited the proliferation of prostate cancer cells, promoted apoptosis, and enhanced the sensitivity of prostate cancer cells to radiation therapy.

In recent years, FBP1's role in tumors has received increasing attention. The expression of the FBP1 gene is absent in a variety of tumors including colon cancer, liver cancer, lung cancer, and gastric cancer, and is associated with poor prognosis in cancer patients [11-15]. The reason for this phenomenon is that FBP1 is related to the Warburg effect of tumor cells, and the downregulation of FBP can promote the survival and development of tumors by enhancing the Warburg effect. FBP1 acts as a tumor suppressor by inhibiting the glycolysis of cells and inhibiting the growth of tumor cells. However, there are contradictory reports in breast cancer. Li et al. analyzed the Oncominde data and found that the FBP1 expression level in all types of breast cancer is higher than that in adjacent tissues. Interfering with FBP1 in MCF-7 and BT474 cells can inhibit the level of glycolysis in breast cancer cells. Mechanism studies have found that FBP1 can negatively regulate $\mathrm{Wnt} / \beta$-Catenin to play an oncogene function [11]. In addition, Chen et al. found that the expression level of FBP1 in breast cancer tissues with brain metastasis was significantly higher than that of local breast cancer tissues and inhibited the expression of FBP and inhibited brain metastasis of breast cancer. Overexpression of FBP1 can increase the level of gluconeogenesis to accommodate the low glucose environment in the metastatic space of the metastases [16]. In this present study, we found that FBP1 is highly expressed in prostate cancer, and knockdown of FBP1 enhances radiosensitivity. Therefore, FBP1 is an oncogene in prostate cancer. The function of FBP1 is tissue and cell specific and is a tumor suppressor gene in intestinal cancer, liver cancer, lung cancer, and gastric cancer, and an oncogene in breast cancer and prostate cancer.

In this study, further experiments confirmed that FBP1 knockdown could activate autophagy mediated by the AMPK-mTOR signaling pathway. Autophagy is a protective mechanism of cells, it can stabilize the intracellular environment when the cells are under stress and provide the necessary energy support for the cells to help to overcome stress [17]. As a common physical toxic stress, radiation therapy can play a role in treating tumors by inducing gene mutations, chromosomal mutations or directly killing cancer cells [18]. Autophagy, also known as type II programmed cell death, is a caspase-independent reversible cell death pathway [19]. Therefore, autophagy induced by cancer cells during radiotherapy often increases cancer cell radiosensitivity to obtain a better therapeutic effect. In lung cancer, both in vitro and in vivo experiments have confirmed that increasing the autophagy level of cancer cells can enhance the sensitivity of cells to radiation [20-22]; the latest report shows that ionizing radiation induces autophagy in colorectal cancer cells, promoting cancer cell death [23]; in prostate cancer cells, radiation can induce autophagy, and combined use of autophagic inhibitors can affect cell survival [24]. Further experiments have shown that activation of the intracellular autophagy pathway can increase the cells' radiosensitivity [10]. Therefore, autophagy plays an important role in regulating the radiation sensitivity of tumor cells [25]. In this study, the knockdown of FBP1 was found to enhance radiation-induced autophagy and promote prostate cancer cell death. Moreover, the inhibition of the AMPK-mTOR signaling pathway reverses the FBP1 knockdown-mediated autophagy and apoptosis, as well as radiosensitization.

In conclusion, this study clarified that FBP1 is an oncogene in prostate cancer, and the main mechanism for knockdown of FBP1 to enhance radiosensitivity is to enhance autophagy mediated by the AMPK-mTOR signaling pathway. Therefore, FBP1 may be a potential target for enhancing prostate cancer radiotherapy.

Acknowledgments: This work was supported by Research of Development and Reform commission of Hunan Province [2016] (No. 65).

\section{References}

[1] SIEGEL R, NAISHADHAM D, JEMAL A. Cancer statistics, 2013. CA Cancer J Clin 2013; 63: 11-30. https://doi. org/10.3322/caac. 21166

[2] WANG LY, CUI JJ, ZHU T, SHAO WH, ZHAO Y et al. Biomarkers identified for prostate cancer patients through genome-scale screening. Oncotarget 2017; 8: 92055-92063. https://doi.org/10.18632/oncotarget.20739

[3] CENTER MM, JEMAL A, LORTET-TIEULENT J, WARD E, FERLAY J et al. International variation in prostate cancer incidence and mortality rates. Eur Urol 2012; 61: 1079-1092. https://doi.org/10.1016/j.eururo.2012.02.054

[4] BASTIAN PJ, BOORJIAN SA, BOSSI A, BRIGANTI A, HEIDENREICH A et al. High-risk prostate cancer: from definition to contemporary management. Eur Urol 2012; 61: 1096-1106. https://doi.org/10.1016/j.eururo.2012.02.031

[5] KEILER L, DOBBINS D, KULASEKERE R, EINSTEIN D. Tomotherapy for prostate adenocarcinoma: a report on acute toxicity. Radiother Oncol 2007; 84: 171-176. https:// doi.org/10.1016/j.radonc.2007.07.012 
[6] LI Y, TANG Z, QI L, CHEN Z, LI D et al. [Analysis of influential factors for prostate biopsy and establishment of logistic regression model for prostate cancer]. Zhong Nan Da Xue Xue Bao Yi Xue Ban 2015; 40: 651-656. https://doi. org/10.11817/j.issn.1672-7347.2015.06.013

[7] TIMSON DJ. Fructose 1,6-bisphosphatase: getting the message across. Biosci Rep 2019; 39: BSR20190124. https://doi. org/10.1042/BSR20190124

[8] BARTRONS R, SIMON-MOLAS H, RODRIGUEZ-GARCIA A, CASTANO E, NAVARRO-SABATE A et al. Fructose 2,6-Bisphosphate in Cancer Cell Metabolism. Front Oncol 2018; 8: 331. https://doi.org/10.3389/fonc.2018.00331

[9] LI B, QIU B, LEE DS, WALTON ZE, OCHOCKI JD et al. Fructose-1,6-bisphosphatase opposes renal carcinoma progression. Nature 2014; 513:251-255. https://doi.org/10.1038/ nature 13557

[10] SHANG ZF, WEI Q, YU L, HUANG F, XIAO BB et al. Suppression of PC-1/PrLZ sensitizes prostate cancer cells to ionizing radiation by attenuating DNA damage repair and inducing autophagic cell death. Oncotarget 2016; 7: 6234062351. https://doi.org/10.18632/oncotarget.11470

[11] LI K, YING M, FENG D, DU J, CHEN S et al. Fructose1,6-bisphosphatase is a novel regulator of Wnt/beta-Catenin pathway in breast cancer. Biomed Pharmacother 2016; 84: 1144-1149. https://doi.org/10.1016/j.biopha.2016.10.050

[12] JIN X, PAN Y, WANG L, ZHANG L, RAVICHANDRAN $\mathrm{R}$ et al. MAGE-TRIM28 complex promotes the Warburg effect and hepatocellular carcinoma progression by targeting FBP1 for degradation. Oncogenesis 2017; 6: e312. https:// doi.org/10.1038/oncsis.2017.21

[13] YANG J, WANG C, ZHAO F, LUO X, QIN M et al. Loss of FBP1 facilitates aggressive features of hepatocellular carcinoma cells through the Warburg effect. Carcinogenesis 2017; 38: 134-143. https://doi.org/10.1093/carcin/bgw109

[14] LIU GM, LI Q, ZHANG PF, SHEN SL, XIE WX et al. Restoration of FBP1 suppressed Snail-induced epithelial to mesenchymal transition in hepatocellular carcinoma. Cell Death Dis 2018; 9: 1132. https://doi.org/10.1038/s41419018-1165-X

[15] LIU X, WANG X, ZHANG J, LAM EK, SHIN VY et al. Warburg effect revisited: an epigenetic link between glycolysis and gastric carcinogenesis. Oncogene 2010; 29: 442-450. https://doi.org/10.1038/onc.2009.332
[16] CHEN J, LEE HJ, WU X, HUO L, KIM SJ et al. Gain of glucose-independent growth upon metastasis of breast cancer cells to the brain. Cancer Res 2015; 75: 554-565. https://doi. org/10.1158/0008-5472.CAN-14-2268

[17] REN F, SHU G, LIU G, LIU D, ZHOU J et al. Knockdown of p62/sequestosome 1 attenuates autophagy and inhibits colorectal cancer cell growth. Mol Cell Biochem 2014; 385: 95-102. https://doi.org/10.1007/s11010-013-1818-0

[18] YAN Y, CHEN X, WANG X, ZHAO Z, HU W et al. The effects and the mechanisms of autophagy on the cancer-associated fibroblasts in cancer. J Exp Clin Cancer Res 2019; 38: 171. https://doi.org/10.1186/s13046-019-1172-5

[19] XIE Y, KANG R, SUN X, ZHONG M, HUANG J et al. Posttranslational modification of autophagy-related proteins in macroautophagy. Autophagy 2015; 11: 28-45. https://doi.org /10.4161/15548627.2014.984267

[20] KIM KW, HWANG M, MORETTI L, JABOIN JJ, CHA YI et al. Autophagy upregulation by inhibitors of caspase-3 and mTOR enhances radiotherapy in a mouse model of lung cancer. Autophagy 2008; 4: 659-668. https://doi.org/10.4161/ auto.6058

[21] MENG MB, WANG HH, GUO WH, WU ZQ, ZENG XL et al. Targeting pyruvate kinase M2 contributes to radiosensitivity of non-small cell lung cancer cells in vitro and in vivo. Cancer Lett 2015; 356: 985-993. https://doi.org/10.1016/j. canlet.2014.11.016

[22] LI Y, LIU F, WANG Y, LI D, GUO F et al. Rapamycin-induced autophagy sensitizes A549 cells to radiation associated with DNA damage repair inhibition. Thorac Cancer 2016; 7: 379-386. https://doi.org/10.1111/1759-7714.12332

[23] CLASSEN F, KRANZ P, RIFFKIN H, POMPSCH M, WOLF A et al. Autophagy induced by ionizing radiation promotes cell death over survival in human colorectal cancer cells. Exp Cell Res 2019; 374: 29-37. https://doi.org/10.1016/j. yexcr.2018.11.004

[24] CHENG X, WANG ZH, PENG M, HUANG ZC, YI L et al. The role of radical prostatectomy and definitive external beam radiotherapy in combined treatment for highrisk prostate cancer: a systematic review and meta-analysis. Asian J Androl. 2019. https://doi.org/10.4103/aja.aja_111_19

[25] CHEN FZ, ZHAO XK. Prostate cancer: current treatment and prevention strategies. Iran Red Crescent Med J 2013; 15 : 279-284. https://doi.org/10.5812/ircmj.6499 